\title{
Standard model anomalies in curved space-time with torsion
}

\author{
Antonio Dobado and Antonio L. Maroto \\ Departamento de Física Teórica, Universidad Complutense de Madrid, 28040 Madrid, Spain
}

(Received 4 August 1995; revised manuscript received 11 March 1996)

\begin{abstract}
Using the Fujikawa and the heat-kernel methods we make a complete and detailed computation of the global, gauge, and gravitational anomalies in the standard model defined on a curved space-time with torsion. We find new contributions coming from the curvature and the torsion to the leptonic number anomaly (so that $B$ - $L$ is not conserved anymore) to the $\mathrm{U}(1)_{Y}$ gauge and to the mixed $\mathrm{U}(1)_{Y}$-gravitational anomalies. However, neither gauge nor gravitational new anomaly cancellation conditions for the hypercharges arise. [S0556-2821(96)05020-5]

PACS number(s): $04.62+\mathrm{v}, 11.30 . \mathrm{Fs}$
\end{abstract}

\section{INTRODUCTION}

Our knowledge of fundamental interactions confirmed experimentally can be summarized roughly in the standard model (SM) and classical gravitation. In other words, any phenomenon ever observed can, in principle, be accommodated in the SM formulated in a curved space-time background. Of course there are many reasons to think that this is not the final theory (provided such a thing exists at all), but at least it is the minimal one compatible with all the experimental data.

For the above reason the proper formulation of the SM in curved space-time has become an important matter. The problem of defining a quantum field theory in curved spacetime has been considered in detail in the literature some time ago (see [1] for a review). Concerning the SM in particular, the most important property is that it is a chiral gauge theory based on the gauge group $\mathrm{SU}(3)_{C} \times \mathrm{SU}(2)_{L} \times \mathrm{U}(1)_{Y}$. Then, the matter (i.e., quarks and leptons) being described by fermionic fields, one is forced to introduce vierbeins and connections on the space-time manifold. As is well known, once a metric is given, there is a unique connection which is metric compatible and torsion-free, namely, the Levi-Civita connection defined by the Christoffel symbols. In fact this was the connection considered by Einstein in his original formulation of general relativity (GR). However, one can also consider the vierbein and the connection as independent structures. In this case, if one starts from the standard EinsteinHilbert action, one may find again the Christoffel symbols for the connection as a solution of an equation of motion together with the Einstein field equations for the metric $(\mathrm{Pa}-$ latini formalism). However, quantum effects or modifications of the action obtained for example by adding higher derivatives terms to the Einstein-Hilbert action could produce torsion. In addition, fermions give a nonzero contribution to the torsion (see, for example, [2]). Finally, most of the extensions of GR introduce the vierbein and the connection as independent entities and this will be our approach in the following. Nevertheless, we will keep the metric compatibility condition in order to have a geometrical meaning for the connection. This condition is equivalent to consider the connection as a $\mathrm{SO}(4)$ or $\mathrm{SO}(3,1)$ Lie-algebra-valued one-form (for Euclidean or Lorentzian signature, respectively).

Thus, in this paper we will address the problem of defin- ing properly the SM as a quantum field theory in the presence of a classical space-time with torsion. As is well known, theories with chiral fermions such as the SM are potentially plagued with gauge and gravitational anomalies which can ruin the consistency of the quantum theory even though it is well defined at the classical level. Fortunately, the current assignment of hypercharges for the different SM fermions is done in such a subtle way that all those anomalies exactly cancel, at least when there is no torsion. In addition, there are global classical symmetries which also turn out to be anomalous, giving rise to interesting physical effects such as the nonconservation of the baryonic or leptonic numbers. In this work we will compute all those anomalies in the SM defined on a curved space-time with torsion.

The plan of the paper goes as follows. In Sec. II we introduce the SM in a curved background space-time with torsion. In Sec. III we discuss some technical aspects concerning the computation of different anomalies, including the appropriate versions of the Fujikawa and heat-kernel methods. In Sec. IV we consider the baryon and lepton number anomalies in the presence of torsion. In Sec. $\mathrm{V}$ we compute the anomalies in the gauge $\mathrm{SU}(3)_{C} \times \mathrm{SU}(2)_{L} \times \mathrm{U}(1)_{Y}$ symmetry. In Sec. VI we study the gravitational anomalies as anomalies in the local Lorentz symmetries. In Sec. VII we discuss some of the consequences of our anomaly computations and in particular those concerning the quantization of the electric charge in the framework of the SM. Finally, in Sec. VIII we briefly list the main results of our work.

\section{THE STANDARD MODEL IN CURVED SPACE-TIME WITH TORSION}

Let us start by introducing some notation [3]. We will use the following conventions for the indices: $m, n, \ldots$ will label objects referred to the locally inertial system whereas $\mu, \nu, \ldots$ will denote world indices. The metric tensor is expressed in terms of the vierbein $e_{m}^{\mu}(x)$ as

$$
g^{\mu \nu}(x)=e_{m}^{\mu}(x) e_{n}^{\nu}(x) \eta^{m n}
$$

where $\eta^{m n}=(-,-,-,-$,$) is the Euclidean flat metric. Notice that$ we work in Euclidean space-time, assuming that the Wick rotation has been performed: $x^{0} \rightarrow-i \hat{x}^{4}, x^{i} \rightarrow \hat{x}^{i}, \partial_{0} \rightarrow i \hat{\partial}_{4}$, $\partial_{i} \rightarrow \hat{\partial}_{i}$, where the carets denote Euclidean space-time ob- 
jects. The Euclidean $\gamma$ matrices are defined by $\gamma^{0} \rightarrow \hat{\gamma}^{4}$, $\gamma^{i} \rightarrow i \hat{\gamma}^{i}$, and $\hat{\gamma}_{5}=-\hat{\gamma}_{1} \hat{\gamma}_{2} \hat{\gamma}_{3} \hat{\gamma}_{4}$. (In the following we will work in Euclidean space-time and the carets will be omitted for simplicity).

The Hermitian form of the Dirac Lagrangian in curved space-time, with fermions considered as anticommuting variables reads

$$
\mathcal{L}=\frac{1}{2}\left(\psi^{\dagger} \gamma^{\mu} D_{\mu} \psi-D_{\mu} \psi^{\dagger} \gamma^{\mu} \psi\right),
$$

where $\gamma^{\mu}=e_{m}^{\mu} \gamma^{m}$ and these matrices satisfy $\left\{\gamma^{\mu}, \gamma^{\nu}\right\}=-2 g^{\mu \nu}$. The gauge and Lorentz covariant derivative is defined as usual by

$$
D_{\mu} \psi=\left(\partial_{\mu}+\Omega_{\mu}+A_{\mu}\right) \psi=\left(\hat{\nabla}_{\mu}+A_{\mu}\right) \psi,
$$

where $\Omega_{\mu}=-(i / 2) \hat{\Gamma}_{\mu}^{a b} \Sigma_{a b}$ is the spin connection with $\Sigma_{m n}=(i / 4)\left[\gamma_{m}, \gamma_{n}\right]$ the Hermitian generators of the $\mathrm{SO}(4)$ group in the spinor representation and $A_{\mu}$ denotes the gauge connection. Notice that $\left\{\hat{\Gamma}_{\mu}^{a b}\right\}$ does not have to be a torsionfree Levi-Civita connection, which we will denote $\left\{\Gamma_{\mu}^{a b}\right\}$. In general, if we take a metric connection, i.e., $\hat{\nabla}_{\nu} g_{\alpha \beta}=0$, then it can be written as

$$
\hat{\Gamma}_{\mu}^{a b}=\Gamma_{\mu}^{a b}+e_{\nu}^{a} e^{\lambda b} K_{\mu \lambda}^{\nu},
$$

where $K_{\mu \lambda}^{\nu}$ is known as the contorsion tensor related to the torsion tensor by [4]

$$
K^{\nu \mu \lambda}=\frac{1}{2}\left(T^{\nu \mu \lambda}+T^{\mu \nu \lambda}+T^{\lambda \nu \mu}\right) .
$$

Using the decomposition in Eq. (4) we can write the Dirac Lagrangian in Eq. (2) (without gauge fields) in terms of the usual Levi-Civita connection plus an additional term depending on the torsion [5]:

$$
\begin{aligned}
\mathcal{L}= & \frac{1}{2}\left[\psi^{\dagger} \gamma^{\mu}\left(\partial_{\mu}-\frac{i}{2} \hat{\Gamma}_{\mu}^{a b} \Sigma_{a b}\right) \psi\right. \\
& \left.-\left(\partial_{\mu} \psi^{\dagger}+\frac{i}{2} \hat{\Gamma}_{\mu}^{a b} \psi^{\dagger} \Sigma_{a b}\right) \gamma^{\mu} \psi\right] \\
= & \psi^{\dagger} \gamma^{\mu}\left(\partial_{\mu}-\frac{i}{2} \hat{\Gamma}_{\mu}^{a b} \Sigma_{a b}+\frac{1}{2} T_{\mu}\right) \psi \\
= & \psi^{\dagger} \gamma^{\mu}\left(\partial_{\mu}-\frac{i}{2} \Gamma_{\mu}^{a b} \Sigma_{a b}-\frac{1}{8} S_{\mu} \gamma_{5}\right) \psi,
\end{aligned}
$$

where $S_{\alpha}=\epsilon_{\mu \nu \lambda \alpha} T^{\mu \nu \lambda}$ and $T_{\mu}=T_{\lambda \mu}^{\lambda}=K_{\lambda \mu}^{\lambda}$. Note that, with this definition, $S_{\mu}$ is the axial part of the torsion tensor. In conclusion, the Lagrangian for Dirac fermions in a curved space-time with torsion is that of a fermion in a curved space-time without torsion plus an axial interaction with $S_{\mu}$. This similarity with axial gauge couplings will simplify the computation of anomalies when using the well-known heatkernel expansion in curved space-time. However, there is a difference between the axial coupling of torsion and the axial couplings of gauge fields. While the latter breaks the Hermiticity of the Dirac operator, the former does not.

Let us use Eq. (6) for the particular case of the SM matter sector with one family of massless fermions and without considering the Yukawa couplings to the Higgs field:

$$
\mathcal{L}_{m}=Q^{\dagger} \not D^{Q} Q+L^{\dagger} \not D^{L} L,
$$

where the Dirac operators for quarks and leptons are defined as

$$
\begin{gathered}
i I D^{Q}=i \gamma^{\mu}\left(\partial_{\mu}+\mathbf{\Omega}_{\mu}^{Q}+\mathbf{G}_{\mu}+\mathbf{W}_{\mu}^{Q} P_{L}+\mathbf{B}_{\mu}^{Q}+\mathbf{S}_{\mu}^{Q} \gamma_{5}\right), \\
i D^{L}=i \gamma^{\mu}\left(\partial_{\mu}+\mathbf{\Omega}_{\boldsymbol{\mu}}^{\mathbf{L}}+\mathbf{W}_{\mu}^{L} P_{L}+\mathbf{B}_{\mu}^{L}+\mathbf{S}_{\mu}^{L} \gamma_{5}\right) .
\end{gathered}
$$

Here we have organized the matter fields in doublets, so that for the first family we have

$$
Q=\left[\begin{array}{l}
u \\
d
\end{array}\right], \quad L=\left[\begin{array}{l}
\nu \\
e
\end{array}\right] .
$$

Their left-handed components $Q_{L}$ and $L_{L}$ are $\mathrm{SU}(2)_{L}$ doublets, whereas each right-handed component, $Q_{R}$ and $L_{R}$, is an $\mathrm{SU}(2)_{L}$ singlet. In addition, the $u$ and $d$ quarks are $\mathrm{SU}(3)_{C}$ triplets. The gauge fields appearing in the operators are gluons, corresponding to the $\mathrm{SU}(3)_{C}$ group, that we will denote by $\mathbf{G}_{\mu}=-i g_{S} G_{\mu}^{a} \Lambda^{a}$ with $\Lambda^{a}$ the Gell-Mann matrices, $W$ bosons, corresponding to the $\mathrm{SU}(2)_{L}$ symmetry, that we will write as $\mathbf{W}_{\mu}^{Q, L}=-i g W_{\mu}^{a} T^{a}$ with the $T^{a}$ generators in the appropriate representation; finally there is also the hypercharge boson

$$
\mathbf{B}_{\mu}^{Q, L}=i g^{\prime} B_{\mu}\left(P_{L} \mathbf{y}_{L}^{Q, L}+P_{R} \mathbf{y}_{R}^{Q, L}\right)
$$

with the hypercharge matrices defined as

$$
\mathbf{y}_{L}^{Q, L}=\left(\begin{array}{cc}
y_{L}^{u, \nu} & \\
& y_{L}^{d, e}
\end{array}\right), \quad \mathbf{y}_{R}^{Q, L}=\left(\begin{array}{ll}
y_{R}^{u, \nu} & \\
& y_{R}^{d, e}
\end{array}\right) .
$$

We should stress that the $\left(i \not D^{Q}\right)$ and $\left(i \not D^{L}\right)$ operators are not Hermitian, due to the chiral couplings of $\mathrm{SU}(2)_{L}$ and hypercharge fields. Thus the adjoint operators are

$$
\begin{gathered}
\left(i D^{Q}\right)^{\dagger}=i \gamma^{\mu}\left(\partial_{\mu}+\mathbf{\Omega}_{\mu}^{Q}+\mathbf{G}_{\mu}+\mathbf{W}_{\mu}^{Q} P_{R}+\overline{\mathbf{B}}_{\mu}^{Q}+\mathbf{S}_{\mu}^{Q} \gamma_{5}\right), \\
\left(i \not D^{L}\right)^{\dagger}=i \gamma^{\mu}\left(\partial_{\mu}+\overline{\mathbf{\Omega}}_{\mu}^{L}+\mathbf{W}_{\mu}^{L} P_{R}+\overline{\mathbf{B}}_{\mu}^{L}+\overline{\mathbf{S}}_{\mu}^{L} \gamma_{5}\right),
\end{gathered}
$$

where

$$
\overline{\mathbf{B}}_{\mu}^{Q, L}=i g^{\prime} B_{\mu}\left(P_{R} \mathbf{y}_{L}^{Q, L}+P_{L} \mathbf{y}_{R}^{Q, L}\right) .
$$

Notice that, since there is no right neutrino, the spin connection is written as follows for leptonic operators:

$$
\begin{gathered}
\boldsymbol{\Omega}_{\mu}^{L}=-\frac{i}{2} \Gamma_{\mu}^{a b}\left(\begin{array}{ll}
P_{L} \Sigma_{a b} & \\
& \Sigma_{a b}
\end{array}\right), \\
\overline{\mathbf{\Omega}}_{\mu}^{L}=-\frac{i}{2} \Gamma_{\mu}^{a b}\left(\begin{array}{ll}
P_{R} \Sigma_{a b} & \\
& \Sigma_{a b}
\end{array}\right)
\end{gathered}
$$

for the same reason, the torsion terms are

$$
\begin{aligned}
& \mathbf{S}_{\mu}^{L} \gamma_{5}=-\frac{1}{8} S_{\mu}\left(\begin{array}{ll}
P_{L} \gamma_{5} & \\
& \gamma_{5}
\end{array}\right), \\
& \overline{\mathbf{S}}_{\mu}^{L} \gamma_{5}=-\frac{1}{8} S_{\mu}\left(\begin{array}{ll}
P_{R} \gamma_{5} & \\
& \gamma_{5}
\end{array}\right) .
\end{aligned}
$$


Finally, it is also important to note that in Eq. (6) we have written the minimal Lagrangian for fermions on a curved space-time with torsion. As we will see later, the introduction of nonminimal terms could yield new contributions to the SM anomalies.

\section{THE HEAT KERNEL FOR THE STANDARD MODEL OPERATORS}

There are several techniques proposed for the computation of anomalies in the literature. For our purposes here the most appropriate is to use the functional methods that were first introduced by Fujikawa [6] in a flat space-time and later extended to curved space-time by Yajima [7]. According to these methods, anomalies arise as Jacobian determinants in the functional integral. These Jacobians are divergent objects and have to be regularized using one of the existing approaches. One possibility is to use the Fujikawa method regularizing the Jacobian by means of a Gaussian cutoff. Thus, for instance, for an axial transformation we would write

$$
\left[d \psi d \psi^{\dagger}\right] \rightarrow\left[d \psi d \psi^{\dagger}\right] \exp \left(-2 \int d^{4} x \sqrt{g} i \alpha(x) A(x)\right),
$$

where the anomaly $A(x)$ appearing in the (regularized) Jacobian reads

$$
\begin{aligned}
A_{\text {reg }} & =\lim _{t \rightarrow \infty} \sum_{n} \phi_{n}^{\dagger} \gamma_{5} \exp \left(-\frac{\lambda_{n}^{2}}{t}\right) \phi_{n} \\
& =\lim _{t \rightarrow \infty} \sum_{n} \phi_{n}^{\dagger} \gamma_{5} \exp \left(-\frac{(i \not D)^{2}}{t}\right) \phi_{n} \\
& =\lim _{t \rightarrow \infty} \operatorname{tr} \frac{t^{2}}{(4 \pi)^{2}} \gamma_{5} \sum_{n=0}^{\infty} \frac{a_{n}(x)}{t^{n}},
\end{aligned}
$$

where $\lambda_{n}$ are the eigenvalues of the Hermitian operator of the theory $(i \not D), \phi_{n}$ are its eigenvectors, and in the last step we have performed the Seeley-De Witt expansion for the heat kernel. In general, the above expression is divergent in the $t \rightarrow \infty$ limit, due to the first two terms, and a certain renormalization prescription will be needed to obtain a finite result. However, it may happen, as in the case of theories with only vector gauge couplings, that those potentially divergent terms vanish yielding a finite value for $A_{\text {reg }}$.

An alternative point of view which provides a finite result for the anomaly without introducing any ad hoc regularizing operator is the $\zeta$-function regularization prescription [8]. In this case the transformation Jacobian is defined as the quotient between the effective action and the transformed effective action, both regularized using $\zeta$-function regularization. Thus, for instance, for the case of the axial transformations considered before,

$$
\operatorname{det} \not D=J \operatorname{det}\left(e^{\gamma_{5} \alpha(x)} \not D e^{\gamma_{5} \alpha(x)}\right),
$$

where $J$ is the Jacobian of the symmetry transformation. With this definition the Jacobian is finite since it is the quotient of two regularized objects. In the case of Hermitian
Dirac operators this prescription gives the same result as the Fujikawa method (once the divergent pieces have been removed). However, this is not so for non-Hermitian operators, for which several regularization operators can be chosen in the Fujikawa approach [9] leading in general to different results. Thus, for instance, one can split the Lagrangian and the integration measure in their left and right components:

$$
\begin{gathered}
{\left[d \psi d \psi^{\dagger}\right] \rightarrow\left[d \psi_{R}^{\dagger} d \psi_{L}^{\dagger} d \psi_{R} d \psi_{L}\right],} \\
\psi^{\dagger} \not D \psi=\psi_{R}^{\dagger} \not D_{L} \psi_{L}+\psi_{L}^{\dagger} D_{R} \psi_{R} .
\end{gathered}
$$

For the non-Hermitian SM Dirac operators in Eq. (8) without torsion (the torsion term is written between brackets) the left and right Dirac quark operators

$$
\begin{gathered}
i \not D_{L}^{Q}=i \gamma^{\mu}\left[\partial_{\mu}+\mathbf{\Omega}_{\mu}^{Q}+\mathbf{G}_{\mu}+\mathbf{W}_{\mu}^{Q}+\mathbf{B}_{L \mu}^{Q}\left(-\mathbf{S}_{\mu}^{Q}\right)\right], \\
i \not D_{R}^{Q}=i \gamma^{\mu}\left[\partial_{\mu}+\mathbf{\Omega}_{\mu}^{Q}+\mathbf{G}_{\mu}+\mathbf{B}_{R \mu}^{Q}\left(+\mathbf{S}_{\mu}^{Q}\right)\right]
\end{gathered}
$$

are Hermitian (the same is true for leptons). Thus, it is possible to regularize separately each piece of the anomaly. However, the torsion term breaks the Hermiticity of these operators and therefore this method does not seem to be suitable in presence of torsion. In spite of this fact, one can always rotate $S_{\mu} \rightarrow i S_{\mu}$ [10]. This makes the operators Hermitian and then, at the end of the calculation, one can undo the rotation. Such a procedure has proved to be useful in theories with axial gauge couplings and yields the so called consistent anomaly. However for torsion it can be seen that, calculating in this way, one finds inconsistent results since there would not be any choice of hypercharges in the SM to cancel all the gauge anomalies.

Another regularization method [9], also in the Fujikawa approach, which does not suffer from these inconsistencies is to regularize separately the pieces in the anomaly coming from the transformation of $\psi$ and $\psi^{\dagger}$. For that purpose, our first step will be to build the following two Hermitian operators which preserve all the gauge symmetries:

$$
\begin{aligned}
& H_{\psi}=(i \not D)^{\dagger}(i \not D), \\
& H_{\psi^{\dagger}}=(i \not D)(i \not D)^{\dagger} .
\end{aligned}
$$

Then the Hermiticity ensures that their corresponding eigenfunctions form a complete set:

$$
\begin{aligned}
& H_{\psi} \phi_{n}=\lambda_{n}^{2} \phi_{n}, \\
& H_{\psi^{\dagger}} \xi_{n}=\lambda_{n}^{2} \xi_{n} .
\end{aligned}
$$

Now we expand $\psi$ and $\psi^{\dagger}$ in terms of eigenfunctions of $H_{\psi}$ and $H_{\psi^{\dagger}}$, respectively, that is, $\psi=\sum_{n} a_{n} \phi_{n}, \quad \psi^{\dagger}$ $=\Sigma_{n} \bar{b}_{n} \xi_{n}^{\dagger}$. Under an infinitesimal transformation such as

$$
\begin{gathered}
\psi \rightarrow \psi-i \alpha^{a}(x) T^{a} \psi, \\
\psi^{\dagger} \rightarrow \psi^{\dagger}+i \psi^{\dagger} \alpha^{a}(x) T^{a},
\end{gathered}
$$

where $T^{a}$ are the generators of the given group (eventually including some chiral projector), the integration measure changes as 
$\left[d \psi d \psi^{\dagger}\right] \propto\left[d a_{n} d \bar{b}_{n}\right] \rightarrow\left[d a_{n}^{\prime} d \bar{b}_{n}^{\prime}\right]$

$$
=\left[d a_{n} d \bar{b}_{n}\right] \exp \left(\int d^{4} x \sqrt{g} i \alpha^{a}(x) A^{a}(x)\right),
$$

where $A^{a}(x)$ is the anomaly

$$
A^{a}(x)=\sum_{n} \phi_{n}^{\dagger} T^{a} \phi_{n}-\sum_{n} \xi_{n}^{\dagger} T^{a} \xi_{n} .
$$

As it has already been mentioned, we regularize each piece of the anomaly with the corresponding operator:

$A^{a}(x)=\lim _{M \rightarrow \infty}\left(\sum_{n} \phi_{n}^{\dagger} T^{a} e^{-H_{\psi} / M^{2}} \phi_{n}-\sum_{n} \xi_{n}^{\dagger} T^{a} e^{-H_{\psi^{\dagger}} / M^{2}} \xi_{n}\right)$.

In order to obtain a finite result, we have to perform the heat-kernel expansion for the $H_{\psi}$ and $H_{\psi^{\dagger}}$ operators and substract the divergent terms. However, a new difficulty still appears. Although the heat-kernel expansion has been worked out for a wide class of operators even in curved space-time, the coefficients become unmanageable [11] for operators which do not cast the general form

$$
H=D_{\mu} D^{\mu}+X,
$$

where $X$ does not contain derivatives. At first glance, this is not the case of $H_{\psi}$ and $H_{\psi^{\dagger}}$. However, with some algebra we can write them in the desired form [12]:

$$
\begin{aligned}
H_{\psi}= & (i \not D)^{\dagger}(i I D)=D_{\mu} D^{\mu}+\gamma_{5} \mathbf{S}_{; \mu}^{\mu}+2 \mathbf{S}_{\mu} \mathbf{S}^{\mu} \\
& -\frac{1}{4}\left[\gamma^{\mu}, \gamma^{\nu}\right]\left[d_{\mu}, d_{\nu}\right], \\
H_{\psi^{\dagger}}= & (i \not D)(i I D)^{\dagger}=\bar{D}_{\mu} \bar{D}^{\mu}+\gamma_{5} \overline{\mathbf{S}}_{; \mu}^{\mu}+2 \overline{\mathbf{S}}_{\mu} \overline{\mathbf{S}}^{\mu} \\
& -\frac{1}{4}\left[\gamma^{\mu}, \gamma^{\nu}\right]\left[\bar{d}_{\mu}, \bar{d}_{\nu}\right],
\end{aligned}
$$

where

$$
\begin{aligned}
& d_{\mu}^{Q, L}=\partial_{\mu}+\mathbf{\Omega}_{\mu}^{Q, L}+\left(\mathbf{G}_{\mu}\right)+\mathbf{W}_{\mu}^{Q, L} P_{L}+\mathbf{B}_{\mu}^{Q, L}, \\
& \bar{d}_{\mu}^{Q, L}=\partial_{\mu}+\overline{\mathbf{\Omega}}_{\mu}^{Q, L}+\left(\mathbf{G}_{\mu}\right)+\mathbf{W}_{\mu}^{Q, L} P_{R}+\overline{\mathbf{B}}_{\mu}^{Q, L},
\end{aligned}
$$

and

$$
\begin{aligned}
& D_{\mu}^{Q, L}=d_{\mu}^{Q, L}-\frac{1}{2} \gamma_{5}\left[\gamma_{\mu}, \gamma^{\nu}\right] \mathbf{S}_{\nu}^{Q, L}, \\
& \bar{D}_{\mu}^{Q, L}=\bar{d}_{\mu}^{Q, L}-\frac{1}{2} \gamma_{5}\left[\gamma_{\mu}, \gamma^{\nu}\right] \overline{\mathbf{S}}_{\nu}^{Q, L} .
\end{aligned}
$$

The gluonic terms written in parentheses are absent in the leptonic operators and $\overline{\mathbf{B}}_{\mu}^{Q, L}, \mathbf{\Omega}_{\mu}^{Q, L}$, and $\overline{\mathbf{S}}_{\nu}^{Q, L}$ have been defined in Eqs. (13), (14), and (15). Therefore, removing the divergent $a_{1}(x)$ coefficient we obtain, for the anomaly,

$$
A^{a}(x)=\frac{1}{(4 \pi)^{2}} \operatorname{tr}\left\{T^{a}\left[a_{2}\left(H_{\psi}, x\right)-a_{2}\left(H_{\psi^{\dagger}}, x\right)\right]\right\},
$$

where the second coefficient in the heat-kernel expansion in curved space-time has been worked out in different references $[7,11,12]$ using different methods and in our case reads (for quarks or leptons)

$$
\begin{aligned}
a_{2}\left(H_{\psi}, x\right)= & \frac{1}{12}\left[D_{\mu}, D_{\nu}\right]\left[D^{\mu}, D^{\nu}\right]+\frac{1}{6}\left[D_{\mu},\left[D^{\mu}, X\right]\right] \\
& +\frac{1}{2} X^{2}-\frac{1}{6} R X-\frac{1}{30} R_{; \mu}^{\mu}+\frac{1}{72} R^{2} \\
& +\frac{1}{180}\left(R_{\mu \nu \rho \sigma} R^{\mu \nu \rho \sigma}-R_{\mu \nu} R^{\mu \nu}\right)
\end{aligned}
$$

and

$$
\begin{aligned}
a_{2}\left(H_{\psi^{\dagger}}, x\right)= & \frac{1}{12}\left[\bar{D}_{\mu}, \bar{D}_{\nu}\right]\left[\bar{D}^{\mu}, \bar{D}^{\nu}\right]+\frac{1}{6}\left[\bar{D}_{\mu},\left[\bar{D}^{\mu}, \bar{X}\right]\right] \\
& +\frac{1}{2} \bar{X}^{2}-\frac{1}{6} \overline{R X}-\frac{1}{30} \bar{R}_{; \mu}^{\mu}+\frac{1}{72} \bar{R}^{2} \\
& +\frac{1}{180}\left(\bar{R}_{\mu \nu \rho \sigma} \bar{R}^{\mu \nu \rho \sigma}-\bar{R}_{\mu \nu} \bar{R}^{\mu \nu}\right)
\end{aligned}
$$

where, according to Eq. (28),

$$
\begin{aligned}
& X=\gamma_{5} \mathbf{S}_{; \mu}^{\mu}+2 \mathbf{S}_{\mu} \mathbf{S}^{\mu}-\frac{1}{4}\left[\gamma^{\mu}, \gamma^{\nu}\right]\left[d_{\mu}, d_{\nu}\right], \\
& \bar{X}=\gamma_{5} \overline{\mathbf{S}}_{; \mu}^{\mu}+2 \overline{\mathbf{S}}_{\mu} \overline{\mathbf{S}}^{\mu}-\frac{1}{4}\left[\gamma^{\mu}, \gamma^{\nu}\right]\left[\bar{d}_{\mu}, \bar{d}_{\nu}\right] .
\end{aligned}
$$

The explicit expression for the commutators in Eqs. (32) and (33) can be written as

$$
\begin{aligned}
{\left[D_{\mu}^{Q, L}, D_{\nu}^{Q, L}\right]=} & \mathbf{R}_{\mu \nu}^{Q, L}+\left(\mathbf{G}_{\mu \nu}\right)+\mathbf{W}_{\mu \nu}^{Q, L} P_{L}+\mathbf{B}_{\mu \nu}^{Q, L} \\
& -\mathbf{S}^{\alpha Q, L} \mathbf{S}_{\alpha}^{Q, L}\left[\gamma_{\nu}, \gamma_{\mu}\right]-\left[\gamma_{\nu}, \gamma^{\alpha}\right] \\
& \times\left(\frac{1}{2} \gamma_{5} \mathbf{S}_{\alpha ; \mu}^{Q, L}-\mathbf{S}_{\mu}^{Q, L} \mathbf{S}_{\alpha}^{Q, L}\right)+\left[\gamma_{\mu}, \gamma^{\alpha}\right] \\
& \times\left(\frac{1}{2} \gamma_{5} \mathbf{S}_{\alpha ; \nu}^{Q, L}-\mathbf{S}_{\nu}^{Q, L} \mathbf{S}_{\alpha}^{Q, L}\right)
\end{aligned}
$$

and

$$
\begin{aligned}
{\left[\bar{D}_{\mu}^{Q, L}, \bar{D}_{\nu}^{Q, L}\right]=} & \overline{\mathbf{R}}_{\mu \nu}^{Q, L}+\left(\mathbf{G}_{\mu \nu}\right)+\mathbf{W}_{\mu \nu}^{Q, L} P_{R}+\overline{\mathbf{B}}_{\mu \nu}^{Q, L} \\
& -\overline{\mathbf{S}}^{\alpha Q, L} \overline{\mathbf{S}}_{\alpha}^{Q, L}\left[\gamma_{\nu}, \gamma_{\mu}\right]-\left[\gamma_{\nu}, \gamma^{\alpha}\right] \\
& \times\left(\frac{1}{2} \gamma_{5} \overline{\mathbf{S}}_{\alpha ; \mu}^{Q, L}-\overline{\mathbf{S}}_{\mu}^{Q, L} \overline{\mathbf{S}}_{\alpha}^{Q, L}\right)+\left[\gamma_{\mu}, \gamma^{\alpha}\right] \\
& \times\left(\frac{1}{2} \gamma_{5} \overline{\mathbf{S}}_{\alpha ; \nu}^{Q, L}-\overline{\mathbf{S}}_{\nu}^{Q, L} \overline{\mathbf{S}}_{\alpha}^{Q, L}\right)
\end{aligned}
$$

Notice that for quarks, the torsion and curvature terms are the same either with or without a bar. The gluonic term in brackets is absent in the leptonic case and we have defined

$$
\begin{aligned}
& \mathbf{R}_{\mu \nu}^{L}=-\frac{i}{2} R^{a b}{ }_{\mu \nu}\left(\begin{array}{ll}
P_{L} \Sigma_{a b} & \\
& \Sigma_{a b}
\end{array}\right), \\
& \overline{\mathbf{R}}_{\mu \nu}^{L}=-\frac{i}{2} R^{a b}{ }_{\mu \nu}\left(\begin{array}{ll}
P_{R} \Sigma_{a b} & \\
& \Sigma_{a b}
\end{array}\right) .
\end{aligned}
$$

Once we have a consistent method for computing anomalies in a curved space-time with torsion, let us apply it to the SM anomalies.

\section{ANOMALIES IN THE LEPTONIC AND BARYONIC CURRENTS}

In this section we will calculate the anomalies in the global vector currents $B$ and $L$. Their difference $B-L$ is conserved in flat space-time although separately they are not. However, we will show that in curved space-times the ab- 
sence of right neutrinos implies that, in some sense, gravity couples chirally and thus the anomaly in the leptonic current acquires a gravitational contribution. Nevertheless, these gravitational terms are not present in the baryonic sector, thus yielding the above commented $B-L$ nonconservation.

In order to obtain the anomalous Ward identities related to the leptonic and baryonic numbers, we will consider the following local transformations of quarks and leptons:

$$
\begin{gathered}
\psi \rightarrow \psi+i \alpha(x) \psi, \\
\psi^{\dagger} \rightarrow \psi^{\dagger}-i \psi^{\dagger} \alpha(x) .
\end{gathered}
$$

Note that the classical action would be invariant under these transformations if they were global. The SM fermionic action for a general spin connection reads

$$
\int d^{4} x \sqrt{g} \mathcal{L}_{m}=\int d^{4} x \sqrt{g} \frac{1}{2}\left(\psi^{\dagger} \gamma^{\mu} D_{\mu} \psi-\left(D_{\mu} \psi\right)^{\dagger} \gamma^{\mu} \psi\right),
$$

where we denote by $\psi$ the leptons and quarks. Under the above transformations, the classical action changes as follows:

$$
\int d^{4} x \sqrt{g} \mathcal{L}_{m} \rightarrow \int d^{4} x \sqrt{g}\left[\mathcal{L}_{m}-i \alpha(x) \nabla_{\mu}\left(\psi^{\dagger} \gamma^{\mu} \psi\right)\right],
$$

where we have used integration by parts with the Levi-Civita covariant derivative $\nabla_{\mu}$. On the contrary, the effective action does not change under the transformation since it only affects to fermion fields which are integration variables:

$$
\begin{aligned}
e^{-W[A, \Gamma, e]} & =\int\left[d \psi d \psi^{\dagger}\right] \exp \left(-\int d^{4} x \sqrt{g} \mathcal{L}_{m}\left(\psi, \psi^{\dagger}\right)\right) \\
& =\int\left[d \psi^{\prime} d \psi^{\prime \dagger}\right] \exp \left(-\int d^{4} x \sqrt{g} \mathcal{L}_{m}\left(\psi^{\prime}, \psi^{\prime \dagger}\right)\right) .
\end{aligned}
$$

Now using Eq. (24) (for an Abelian group) and Eq. (40), we obtain for the effective action the expression

$$
\begin{aligned}
e^{-W[A, \Gamma, e]=} & \int\left[d \psi d \psi^{\dagger}\right] \exp \left(-\int d^{4} x \sqrt{g} i \alpha(x) A(x)\right) \\
& \times \exp \left(\int d^{4} x \sqrt{g} i \alpha(x) \nabla_{\mu} j^{\mu}\right) \\
& \times \exp \left(-\int d^{4} x \sqrt{g} \mathcal{L}_{m}\left(\psi, \psi^{\dagger}\right)\right) .
\end{aligned}
$$

Identifying the exponents in Eqs. (41) and (42) we find

$$
A(x)=\nabla_{\mu} j^{\mu} .
$$

Let us recall now the regularized expression for the anomaly derived in the previous section in Eq. (31). Hence, if the transformations in Eq. (38) are applied to quarks, we obtain the anomaly in the baryonic current:

$$
\begin{aligned}
\nabla_{\mu} j_{B}^{\mu}= & \frac{1}{32 \pi^{2}} \epsilon^{\mu \nu \alpha \beta}\left(\frac{g^{2}}{2} W_{\mu \nu}^{a} W_{\alpha \beta}^{a}\right. \\
& \left.+g^{\prime 2} B_{\mu \nu} B_{\alpha \beta} \sum_{u, d}\left(y_{L}^{2}-y_{R}^{2}\right)\right),
\end{aligned}
$$

where the baryonic current is defined in the usual form

$$
j_{B}^{\mu}=\frac{1}{N_{c}} Q^{\dagger} \gamma^{\mu} Q
$$

We see that the result agrees with that of the flat spacetime case. There is no contribution from the curvature nor the torsion.

Following the same steps for leptons, we obtain the anomaly in the leptonic current which reads

$$
\begin{aligned}
\nabla_{\mu} j_{L}^{\mu}= & \frac{1}{32 \pi^{2}}\left\{-\frac{\epsilon^{\alpha \beta \gamma \delta}}{24} R_{\mu \nu \alpha \beta} R_{\gamma \delta}^{\mu \nu}+\frac{\epsilon^{\alpha \beta \gamma \delta}}{48} S_{\beta ; \gamma} S_{\delta ; \alpha}\right. \\
& +\epsilon^{\alpha \beta \gamma \delta}\left(\frac{g^{2}}{2} W_{\gamma \delta}^{a} W_{\alpha \beta}^{a}+g^{\prime 2} B_{\gamma \delta} B_{\alpha \beta} \sum_{\nu, e}\left(y_{L}^{2}-y_{R}^{2}\right)\right) \\
& +\frac{1}{6} \square S_{; \alpha}^{\alpha}+\frac{1}{96}\left(S^{\alpha} S^{\nu} S_{\alpha}\right)_{; \nu} \\
& \left.-\frac{1}{6}\left(R^{\nu \alpha} S_{\alpha}-\frac{1}{2} R S^{\nu}\right)_{; \nu}\right\}
\end{aligned}
$$

where we have defined the leptonic current as

$$
j_{L}^{\mu}=L^{\dagger} \gamma^{\mu} L .
$$

We see that, in this case, due to the absence of right neutrinos, some terms depending on the curvature [13] and the torsion appear in the anomaly as total divergences. It is possible to absorb all the dependence on the torsion by defining the following new current whose divergence gives the same result as in a curved space-time without torsion:

$$
\begin{aligned}
\widetilde{j}_{L}^{\mu}= & j_{L}^{\mu}-\frac{1}{32 \pi^{2}}\left[\frac{1}{6} S_{\alpha}^{; \alpha \mu}+\frac{1}{96} S^{\alpha} S^{\mu} S_{\alpha}\right. \\
& \left.-\frac{1}{6}\left(R^{\mu \alpha} S_{\alpha}-\frac{1}{2} R S^{\mu}\right)+\frac{1}{48} \epsilon^{\mu \beta \gamma \delta} S_{\beta ; \gamma} S_{\delta}\right] .
\end{aligned}
$$

It explicitly depends on the torsion and this fact could have some relevance in the problem of the matter-antimatter asymmetry in the Universe.

Had we assumed the existence of right neutrinos, such terms depending on the curvature and the torsion would have not appeared and $B-L$ would be conserved (as it happens in flat space-time) provided the following relation is satisfied:

$$
\sum_{u, d}\left(y_{L}^{2}-y_{R}^{2}\right)=\sum_{\nu, e}\left(y_{L}^{2}-y_{R}^{2}\right)
$$

which is indeed the case with the usual SM hypercharge assignment. 


\section{GAUGE ANOMALIES}

In this section we will study whether the presence of the curvature and the torsion may introduce new constraints to the hypercharge assignments in the SM.

The matter Lagrangian given in Eq. (7) is invariant under the $\mathrm{SU}(3)_{C} \times \mathrm{SU}(2)_{L} \times \mathrm{U}(1)_{Y}$ gauge transformations, which are given, respectively, by

$$
\begin{gathered}
Q \rightarrow Q-i \theta^{a}(x) \Lambda^{a} Q, \\
Q^{\dagger} \rightarrow Q^{\dagger}+i \theta^{a}(x) Q^{\dagger} \Lambda^{a},
\end{gathered}
$$

for the $\mathrm{SU}(3)_{C}$ transformations for quarks; we also have

$$
\begin{gathered}
\psi \rightarrow \psi-i \theta^{a}(x) T^{a} P_{L} \psi, \\
\psi^{\dagger} \rightarrow \psi^{\dagger}+i \theta^{a}(x) \psi^{\dagger} P_{R} T^{a},
\end{gathered}
$$

for the $\mathrm{SU}(2)_{L}$ transformations of quarks and leptons and, finally, the hypercharge transformation reads

$$
\begin{gathered}
\psi \rightarrow \psi-i \theta(x)\left(\mathbf{y}_{\mathbf{L}} P_{L}+\mathbf{y}_{\mathbf{R}} P_{R}\right) \psi, \\
\psi^{\dagger} \rightarrow \psi^{\dagger}+i \theta(x) \psi^{\dagger}\left(\mathbf{y}_{\mathbf{L}} P_{R}+\mathbf{y}_{\mathbf{R}} P_{L}\right),
\end{gathered}
$$

where $\mathbf{y}_{\mathbf{L}}$ and $\mathbf{y}_{\mathbf{R}}$ are the hypercharge matrices defined in Eq. (11).

In the following we will obtain the expression for the anomalous variation of the effective action for the case of $\mathrm{SU}(3)_{C}$ transformations, being the computation analogous for the other groups. Let us first introduce the notation $\theta=-i \theta^{a} \Lambda^{a}, D_{\mu} \theta=\partial_{\mu} \theta+\left[\mathbf{G}_{\mu}, \theta\right]$. We will also use the definition of the gauge current vacuum expectation value in the presence of the background fields:

$$
\frac{\delta W}{\delta G_{\mu}^{a}}=-i g_{S}\left\langle Q^{\dagger} \gamma^{\mu} \Lambda^{a} Q\right\rangle=-i g_{S}\left\langle j^{\mu a}\right\rangle
$$

We also define $\left\langle j^{\mu}\right\rangle=\left\langle j^{\mu a} \Lambda^{a}\right\rangle$. Under the previously mentioned $\mathrm{SU}(3)_{C}$ transformations the gauge fields change as follows: $\mathbf{G}_{\mu} \rightarrow \mathbf{G}_{\mu}-D_{\mu} \theta$ and the anomalous change in the effective action is given by

$$
W[G-D \theta, \Gamma, e]-W[G, \Gamma, e]=-\int d^{4} x \sqrt{g} i \theta^{b}\left(D_{\mu}\left\langle j^{\mu}\right\rangle\right)^{b},
$$

where we have denoted $D_{\mu}\left\langle j^{\mu}\right\rangle=\nabla_{\mu}\left\langle j^{\mu}\right\rangle+\left[\mathbf{G}_{\mu},\left\langle j^{\mu}\right\rangle\right]$ $=\left(D_{\mu}\left\langle j^{\mu}\right\rangle\right)^{a} \Lambda^{a}$. The change in the integration measure is given in Eq. (24):

$$
A^{a}(x)_{\mathrm{SU}(3)}=\sum_{n}\left(\phi_{n}^{\dagger} \Lambda^{a} \phi_{n}-\xi_{n}^{\dagger} \Lambda^{a} \xi_{n}\right) .
$$

Therefore we can write the transformed effective action as

$$
\begin{aligned}
e^{-W\left[G^{\prime}, \Gamma, e\right]}= & \int\left[d \psi d \psi^{\dagger}\right] \exp \left(-\int d^{4} x \sqrt{g} \mathcal{L}_{m}\right) \\
& \times \exp \left(\int d^{4} x \sqrt{g} \theta^{a}(x) A^{a}(x)\right) .
\end{aligned}
$$

Expanding to first order in $\theta$ and identifying with Eq. (54) we obtain

$$
-\left(D_{\mu}\left\langle j^{\mu}\right\rangle\right)^{a}=A^{a}(x) .
$$

This anomalous Ward identity implies that the nonconservation of the gauge current expectation value is given by the anomaly coefficient. Finally the expression for the anomaly in the $\mathrm{SU}(2)_{L}$ and $\mathrm{U}(1)_{Y}$ currents are given by

$$
\begin{gathered}
A^{a}(x)_{\mathrm{SU}(2)}=\sum_{n}\left(\phi_{n}^{\dagger} P_{L} T^{a} \phi_{n}-\xi_{n}^{\dagger} P_{R} T^{a} \xi_{n}\right), \\
A(x)_{\mathrm{U}(1)}=\sum_{n}\left(\phi_{n}^{\dagger}\left(\mathbf{y}_{\mathbf{L}} P_{L}+\mathbf{y}_{\mathbf{R}} P_{R}\right) \phi_{n}-\xi_{n}^{\dagger}\left(\mathbf{y}_{\mathbf{L}} P_{R}+\mathbf{y}_{\mathbf{R}} P_{L}\right) \xi_{n}\right)
\end{gathered}
$$

We will use the operators $H_{\psi}$ and $H_{\psi^{\dagger}}$ defined in Eq. (28) to regularize each piece of the anomaly separately. The results for the different anomalies are the following.

Anomaly in the $\mathrm{SU}(3)_{C}$ gauge current:

$A_{\mathrm{SU}(3)}^{a}(x)=\frac{1}{(4 \pi)^{2}} \operatorname{tr}\left[\Lambda^{a}\left(a_{2}\left(H_{\psi}, x\right)-a_{2}\left(H_{\psi^{\dagger}}, x\right)\right)\right]$

and for the divergence of the current we have

$$
\left(D_{\mu}\left\langle j^{\mu}\right\rangle\right)^{a}=\frac{1}{32 \pi^{2}} g_{S} g^{\prime} \epsilon^{\mu \nu \alpha \beta} G_{\mu \nu}^{a} B_{\alpha \beta} \sum_{u, d}\left(y_{L}-y_{R}\right) .
$$

This result agrees with that found in flat space-time. There are no new contributions from curvature or torsion. The cancellation condition for this anomaly is given by

$$
\sum_{u, d}\left(y_{L}-y_{R}\right)=0
$$

Anomaly in the $\mathrm{SU}(2)_{L}$ gauge current. Following the same steps as before for the $\mathrm{SU}(2)_{L}$ transformations, we find

$$
A_{\mathrm{SU}(2)}^{a}(x)=\frac{1}{(4 \pi)^{2}} \operatorname{tr}\left[T^{a}\left(a_{2}\left(H_{\psi}, x\right) P_{L}-a_{2}\left(H_{\psi^{\dagger}}, x\right) P_{R}\right)\right] .
$$

The expression for the divergence of the gauge current can be obtained after some algebra and it yields

$$
\left(D_{\mu}\left\langle j^{\mu}\right\rangle\right)^{a}=\frac{1}{32 \pi^{2}} g g^{\prime} \epsilon^{\mu \nu \alpha \beta} W_{\mu \nu}^{a} B_{\alpha \beta}\left(\sum_{u, d} N_{C} y_{L}+\sum_{\nu, e} y_{L}\right) .
$$

We observe that the result is again the same as in flat space-time. All the contributions coming from the curvature or the torsion vanish. The cancellation condition reads in this case 


$$
\sum_{u, d} N_{C} y_{L}+\sum_{\nu, e} y_{L}=0
$$

Anomaly in the $\mathrm{U}(1)_{Y}$ gauge current. Finally, the expression for the anomaly in the $\mathrm{U}(1)_{Y}$ current can be written as

$$
\begin{aligned}
A_{\mathrm{U}(1)}(x)= & \frac{1}{(4 \pi)^{2}} \operatorname{tr}\left[\left(\mathbf{y}_{\mathbf{L}} P_{L}+\mathbf{y}_{\mathbf{R}} P_{R}\right) a_{2}\left(H_{\psi}, x\right)\right. \\
& \left.-\left(\mathbf{y}_{\mathbf{L}} P_{R}+\mathbf{y}_{\mathbf{R}} P_{L}\right) a_{2}\left(H_{\psi^{\dagger}}, x\right)\right] .
\end{aligned}
$$

The final expression for the divergence of the gauge current is now more involved than the non-Abelian cases. The result is

$$
\begin{aligned}
D_{\mu}\left\langle j^{\mu}\right\rangle= & -\frac{1}{32 \pi^{2}}\left\{[ \sum _ { u , d } N _ { C } ( y _ { L } - y _ { R } ) + \sum _ { \nu , e } ( y _ { L } - y _ { R } ) ] \left[-\frac{1}{24} \epsilon^{\alpha \beta \gamma \delta} R_{\mu \nu \alpha \beta} R_{\gamma \delta}^{\mu \nu}+\frac{1}{6} \square S_{; \mu}^{\mu}+\frac{1}{96}\left(S^{\alpha} S^{\nu} S_{\alpha}\right)_{; \nu}\right.\right. \\
& \left.+\frac{1}{48} \epsilon^{\alpha \beta \gamma \delta} S_{\beta ; \gamma} S_{\delta ; \alpha}-\frac{1}{6}\left(R^{\nu \alpha} S_{\alpha}-\frac{1}{2} R S^{\nu}\right)_{; \nu}\right]+\frac{g_{s}^{2}}{2} \epsilon^{\mu \nu \alpha \beta} G_{\mu \nu}^{a} G_{\alpha \beta}^{a} \sum_{u, d}\left(y_{L}-y_{R}\right) \\
& \left.+\frac{g^{2}}{4} \epsilon^{\mu \nu \alpha \beta} W_{\mu \nu}^{a} W_{\alpha \beta}^{a}\left(\sum_{u, d} N_{C} y_{L}+\sum_{\nu, e} y_{L}\right)+g^{\prime 2} \epsilon^{\mu \nu \alpha \beta} B_{\mu \nu} B_{\alpha \beta}\left(\sum_{u, d} N_{C}\left(y_{L}^{3}-y_{R}^{3}\right)+\sum_{\nu, e}\left(y_{L}^{3}-y_{R}^{3}\right)\right)\right\} .
\end{aligned}
$$

Notice the appearance of terms depending on the curvature and the torsion. Also note that the torsion appears only in terms which are four-divergencies and therefore can be removed by adding suitable local counterterms to the Lagrangian which respect the rest of the gauge symmetries [14]. These counterterms read

$$
\Delta \mathcal{L}=-\frac{1}{32 \pi^{2}}\left[\frac{1}{6} B^{\alpha} S_{; \mu \alpha}^{\mu}+\frac{1}{96} B^{\alpha} S^{2} S_{\alpha}+\frac{1}{48} \epsilon^{\alpha \beta \gamma \delta} S_{\beta ; \gamma} S_{\delta} B_{\alpha}-\frac{1}{6} B_{\nu}\left(R^{\nu \alpha} S_{\alpha}-\frac{1}{2} R S^{\nu}\right)\right]\left(\sum_{u, d} N_{C}\left(y_{L}-y_{R}\right)+\sum_{\nu, e}\left(y_{L}-y_{R}\right)\right) .
$$

The new terms that were not present in flat space-time impose a new cancellation condition, namely, the vanishing of the sum of all hypercharges:

$$
\sum_{u, d} N_{C}\left(y_{L}-y_{R}\right)+\sum_{\nu, e}\left(y_{L}-y_{R}\right)=0
$$

which is satisfied by the usual SM hypercharges. On the other hand, we have that the cancellation of the terms already present in flat space-time gives the same conditions as in Eqs. (61) and (64) plus the additional one:

$$
0=\sum_{u, d} N_{C}\left(y_{L}^{3}-y_{R}^{3}\right)+\sum_{\nu, e}\left(y_{L}^{3}-y_{R}^{3}\right)
$$

\section{GRAVITATIONAL ANOMALIES}

In this section we consider the possible violation of local Lorentz symmetry due to quantum effects when chiral fermions are present [15]. We will conclude that whenever Abelian chiral gauge fields are present, as is the case of the hypercharge field, local Lorentz invariance is violated. However, due to the specific hypercharge assignment in the SM this anomaly is exactly cancelled. The condition for the cancellation of the Lorentz anomaly is the same as that of the cancellation of terms depending on the curvature and the torsion in the $\mathrm{U}(1)_{Y}$ anomaly, Eq. (68).

Under local Lorentz transformations the spinor, vierbein, and connection fields present in the matter Lagrangian of the SM, Eq. (7) change as

$$
\begin{gathered}
\psi(x) \rightarrow e^{(i / 2) \epsilon^{m n}(x) \Sigma_{m n}} \psi(x), \\
\psi^{\dagger}(x) \rightarrow \psi^{\dagger}(x) e^{-(i / 2) \epsilon^{m n}(x) \Sigma_{m n},} \\
\epsilon_{\mu}^{a} \rightarrow \epsilon_{\mu}^{a}-\epsilon_{b}^{a}(x) \epsilon_{\mu}^{b}, \\
\Gamma_{\mu}^{a b} \rightarrow \Gamma_{\mu}^{a b}+\epsilon_{c}^{a}(x) \Gamma_{\mu}^{c b}-\epsilon_{c}{ }^{b}(x) \Gamma_{\mu}^{a c}-\partial_{\mu} \epsilon^{a b}(x) .
\end{gathered}
$$

Under these transformations the matter Lagrangian is invariant. However, the effective action changes as

$$
\begin{aligned}
W[A, \Gamma-D \epsilon, e-\epsilon e] \\
=W[A, \Gamma, e]-\int d^{4} x \sqrt{g}\left(\left[-\epsilon_{c}^{a}(x) \Gamma_{\mu}^{c b}+\epsilon_{c}^{b}(x) \Gamma_{\mu}^{a c}\right.\right. \\
\left.\left.\quad+\partial_{\mu} \epsilon^{a b}(x)\right] \frac{\delta W}{\delta \Gamma_{\mu}^{a b}}+\epsilon_{b}^{a}(x) e_{\mu}^{b} \frac{\delta W}{\delta e_{\mu}^{a}}\right)
\end{aligned}
$$

Here we have denoted by $A$ all the gauge fields in the theory. Now, using integration by parts and the antisymmetry of the connection components $\Gamma_{\mu}^{a b}$ in $a$ and $b$, we can rewrite this expression as

$$
\begin{aligned}
& W[A, \Gamma-D \epsilon, e-\epsilon e] \\
& \quad=W[A, \Gamma, e]+\int d^{4} x \sqrt{g} \epsilon_{a b}(x)\left(-\frac{i}{2}\left(D_{\mu}\left\langle j^{\mu}\right\rangle\right)^{a b}-T^{a b}\right),
\end{aligned}
$$


where $T_{a b}=e_{b \mu} \delta W / \delta e_{\mu}^{a}$ is the expectation value of the energy-momentum tensor in presence of the background fields and we have used the following definitions:

$$
\frac{\delta W}{\delta \Gamma_{\mu}^{a b}}=-\frac{i}{4}\left\langle\psi^{\dagger}\left(\gamma^{\mu} \Sigma_{a b}+\Sigma_{a b} \gamma^{\mu}\right) \psi\right\rangle=-\frac{i}{2}\left\langle j_{a b}^{\mu}\right\rangle
$$

with $\left\langle j^{\mu}\right\rangle=\left\langle j_{a b}^{\mu} \Sigma^{a b}\right\rangle$ and $D_{\mu}\left\langle j^{\mu}\right\rangle=\nabla_{\mu}\left\langle j^{\mu}\right\rangle+\left[\Omega_{\mu},\left\langle j^{\mu}\right\rangle\right]$.

In addition, we can calculate the change in the effective action due to the change in the integration measure:

$$
\begin{aligned}
e^{-W\left[A, \Gamma^{\prime}, e^{\prime}\right]}= & \int\left[d \psi d \psi^{\dagger}\right] \exp \left(-\int d^{4} x \sqrt{g} \mathcal{L}_{m}\right) \\
& \times \exp \left(-\frac{i}{2} \int d^{4} x \sqrt{g}\left[\epsilon_{m n}(x) A^{m n}(x)\right]\right),
\end{aligned}
$$

where

$$
A^{m n}(x)=\sum_{n}\left(\phi_{n}^{\dagger \Sigma^{m n}} \phi_{n}-\xi_{n}^{\dagger} \Sigma^{m n} \xi_{n}\right)
$$

Finally, expanding Eq. (74) to first order in $\epsilon$ and identifying the terms in Eq. (72) we find the anomalous identity

$$
A^{m n}(x)=-\left(D_{\mu}\left\langle j^{\mu}\right\rangle\right)^{m n}+i\left(T^{m n}-T^{n m}\right) .
$$

We use the operators $H_{\psi}$ and $H_{\psi^{\dagger}}$ to regularize the first and second terms in Eq. (75), respectively. The result can be expressed as

$$
A_{\mathrm{SO}(4)}^{m n}(x)=\frac{1}{(4 \pi)^{2}} \operatorname{tr}\left[\Sigma^{m n}\left(a_{2}\left(H_{\psi}, x\right)-a_{2}\left(H_{\psi^{\dagger}}, x\right)\right)\right] .
$$

After a lengthy calculation we find the final expression for the Lorentz anomaly:

$$
\begin{aligned}
A^{m n}(x)= & \frac{g^{\prime}}{32 \pi^{2}}\left(\frac{1}{6} \epsilon^{m n a b} R_{\mu \nu a b} B^{\mu \nu}+\frac{1}{6}\left(B_{\alpha}{ }^{n} S^{\alpha ; m}-B_{\alpha}{ }^{m} S^{\alpha ; n}\right)-\frac{1}{24} \epsilon^{m n a b}\left(B_{a c} S^{c} S_{b}+B_{a b} S^{2}\right)-\frac{1}{6} \epsilon^{m n a b} B_{a b} R-\frac{1}{2} S_{; \mu}^{\mu} B^{m n}\right. \\
& \left.-\frac{1}{3} \epsilon^{m n a b} \square B_{a b}+\frac{1}{3}\left(S_{\alpha} B^{\alpha m}\right)^{; n}-\frac{1}{3}\left(S^{m} B^{\mu n}-S^{n} B^{\mu m}\right)_{; \mu}-\frac{1}{3}\left(S_{\alpha} B^{\alpha n}\right) ; m\right)\left(\sum_{u, d} N_{c}\left(y_{L}-y_{R}\right)+\sum_{\nu, \epsilon}\left(y_{L}-y_{R}\right)\right) .
\end{aligned}
$$

Notice that pure gravity terms do not appear. Indeed it has been shown that there are no pure gravitational anomalies in four dimensions [16]. The cancellation condition agrees with that of Eq. (68) which ensures the vanishing of the gravity terms in the $\mathrm{U}(1)_{Y}$ anomaly and, as we have already commented, is satisfied in the SM. Observe also that all the terms depend on the $B_{a b}$ field, which is Abelian, whereas there is no contribution from non-Abelian gauge fields. Some of these terms, such as those being total derivatives, could be eliminated by means of $\mathrm{U}(1)_{Y}$ gauge-invariant counterterms. In principle, the remaining terms could be cancelled by $\mathrm{U}(1)_{Y}$ gauge-noninvariant counterterms and then trade local Lorentz invariance for gauge invariance (see, for instance, [17] for the case without torsion), however in our case it is not obvious which is the form of the counterterms needed for that aim.

Finally, we should stress that the preceding anomaly calculations have been performed with the minimal Lagrangian in Eq. (6). The introduction of nonminimal couplings to torsion or curvature could yield new contributions to the anomalies. In fact, consider the real term $i \psi^{\dagger} \gamma^{\mu} T_{\mu} \psi$ [5]. It is analogous to an Abelian gauge term [like the $\mathrm{U}(1)_{Y}$ ] with the same couplings to the left- and right-handed spinor components (except for the neutrino). From the above results we see that such a term would yield an anomaly in the $\mathrm{SU}(2)_{L}$, $\mathrm{U}(1)_{Y}$ as well as in the local Lorentz symmetry that could not be cancelled by hypercharge assignments.

\section{CHARGE QUANTIZATION IN THE STANDARD MODEL}

In this section we will discuss the consequences of the requirement of the cancellation of the above computed gauge and gravitational anomalies for one family. First of all, we should point out that, at the classical level, gauge invariance of the Yukawa sector of the minimal standard model with a complex Higgs doublet imposes [18] two new constraints: namely,

$$
\begin{gathered}
\frac{1}{2} y_{R}^{d}-\frac{1}{2} y_{R}^{u}=-y_{\phi}, \\
\frac{3}{2} y_{R}^{d}+\frac{3}{2} y_{R}^{u}=y_{\phi},
\end{gathered}
$$

where $y_{\phi}$ is the hypercharge of the Higgs doublet. Therefore, just imposing gauge invariance at the quantum level in flat space-time we obtain five equations. Eqs. (61), (64), (69), and those in Eq. (79) for six unknowns: $y_{L}^{\nu}=y_{L}^{e}, y_{L}^{u}=y_{L}^{d}$, $y_{R}^{e}, y_{R}^{u}, y_{R}^{d}$, and $y_{\phi}$. We can solve this system and obtain all the hypercharges in terms of one of them, for instance, $y_{\phi}$, the result is $y_{L}^{e}=y_{L}^{v}=-y_{\phi}, y_{L}^{u}=y_{L}^{d}=y_{\phi} / 3, y_{R}^{u}=4 y_{\phi} / 3$, and $y_{R}^{d}=-2 y_{\phi} / 3$. Now fixing the electron charge to be -1 it gives the usual hypercharge assignment in the SM. Now, the cancellation condition in Eq. (68) for both the gravitational anomalies and the gravitational terms in the $\mathrm{U}(1)_{Y}$ anomaly does not impose any new constraint on the hypercharges. 
On the other hand, one could have taken a different point of view and, without assuming any specific symmetrybreaking sector, i.e., discarding Eqs. (79), try to fix the hypercharges. In this case, gauge invariance in flat space-time is not enough to fix them and it is necessary to impose the condition in Eq. (68) to obtain a discrete number of solutions. Now we have four equations. Eqs. (61), (64), (69), coming from gauge invariance and Eq. (68) for five unknowns $y_{L}^{\nu}=y_{L}^{e}, y_{L}^{u}=y_{L}^{d}, y_{R}^{e}, y_{R}^{u}, y_{R}^{d}$ [19]. We note that the four equations can be reduced to just one equation for two unknowns: namely,

$$
21\left(y_{R}^{u}\right)^{2} y_{R}^{d}+21\left(y_{R}^{u}\right)^{2} y_{R}^{d}+6\left(y_{R}^{u}\right)^{3}+6\left(y_{R}^{d}\right)^{3}=0 .
$$

This equation, in turn, can be expressed in terms of one variable if $y_{R}^{d} \neq 0$ :

$$
1+\left(\frac{y_{R}^{u}}{y_{R}^{d}}\right)^{3}+\frac{21}{6}\left(\frac{y_{R}^{u}}{y_{R}^{d}}\right)^{2}+\frac{21}{6} \frac{y_{R}^{u}}{y_{R}^{d}}=0
$$

Now it is not difficult to see that there are three real solutions for this equation: $y_{R}^{u} / y_{R}^{d}=-1,-2,-1 / 2$. The rest of hypercharges can be obtained as follows: $y_{L}^{u}$ $=y_{L}^{d}=\left(y_{R}^{u}+y_{R}^{d}\right) / 2, y_{L}^{e}=y_{L}^{\nu}=-3\left(y_{R}^{u}+y_{R}^{d}\right) / 2, y_{R}^{e}=-3\left(y_{R}^{u}\right.$ $\left.+y_{R}^{d}\right)$. Therefore, there are three possible sets of hypercharges (up to a global normalization factor). The first one reads $y_{R}^{u}=-y_{R}^{d}$ and $y_{L}^{u}=y_{L}^{d}=y_{L}^{e}=y_{L}^{\nu}=y_{R}^{e}=0$. This solution together with the usual weak isospin assignment $Q=T_{3}+Y / 2$ implies a chargeless electron and it is the "bizarre" hypercharge assignment obtained in [18].

The second solution, taking the normalization so that the electric charge of the electron is -1 , gives the following values for the hypercharges: $y_{R}^{u}=4 / 3, \quad y_{R}^{d}=-2 / 3$, $y_{L}^{u}=y_{L}^{d}=1 / 3, \quad y_{L}^{e}=y_{L}^{\nu}=-1$, and $y_{R}^{e}=-2$, which is the usual hypercharge assignment in the SM.

The third solution, also taking the same normalization as before, reads $y_{R}^{d}=4 / 3, \quad y_{R}^{u}=-2 / 3, \quad y_{L}^{u}=y_{L}^{d}=1 / 3$, $y_{L}^{e}=y_{L}^{\nu}=-1$, and $y_{R}^{e}=-2$. With the standard weak isospin assignment, the last set leads to different electric charges for the left and right components of the quark fields and therefore to chiral electromagnetism.

To summarize, gauge invariance in flat space-time in the minimal SM with one Higgs doublet is enough to fix all the hypercharges (up to a normalization factor). On the other hand, without assuming any specific symmetry breaking sector, but demanding gauge invariance in curved space-time (or gauge and local Lorentz invariance), we cannot fix the hypercharges, obtaining three possible sets of solutions. Further physical requirements as the existence of a charged electron or vector electromagnetism can be invoked to remove the two unusual solutions in this case.

\section{CONCLUSIONS}

In this work we have carefully computed the different anomalies that appear in the standard model (SM) defined in a classical background space-time with torsion using a welldefined method to regularize the divergencies.

Concerning the anomalies affecting global currents we arrive to the following results. The baryonic current anomaly is not modified by any curvature or torsion term and then it is the same as in flat space-time. However, due to the absence of right-handed neutrinos, the leptonic current anomaly gets new contributions coming from curvature and torsion terms. Therefore, the conservation of the total baryonic minus leptonic number $(B-L)$, which is well known in the SM in flat space-time, is violated when curvature and torsion are present.

The gauge anomalies corresponding to the groups $\mathrm{SU}(3)_{C}$ and $\mathrm{SU}(2)_{L}$ do not get new contributions and then we find the standard conditions for their cancellation in terms of the fermion hypercharges. For the $\mathrm{U}(1)_{Y}$ anomaly we obtain the known contributions from all the SM gauge fields and also new terms depending on the curvature and the torsion. The cancellation of these gauge and gravitational terms gives rise to two more conditions in addition to the other two obtained from the cancellation of the $\mathrm{SU}(3)_{C}$ and $\mathrm{SU}(2)_{L}$ anomalies.

The gravitational anomaly has been computed as a gauge anomaly corresponding to the local (Euclidean) Lorentz group $\mathrm{SO}(4)$. This anomaly has contributions which are products of the hypercharge gauge field and curvature (mixed gauge-gravitational anomalies), hypercharge and torsion and hypercharge alone. This is consistent with the wellknown absence of pure gravitational anomalies in four dimensions. On the other hand, the only condition found on the hypercharges in order to cancel these terms is exactly the same that cancels the curvature and the torsion terms appearing in the $\mathrm{U}(1)_{Y}$ gauge anomaly.

Finally we have dealt with the problem of charge quantization in the SM. It has been shown that including the Higgs sector of the minimal SM and demanding gauge invariance at the quantum level, the hypercharges are fixed, up to normalization. On the other hand, without considering any specific symmetry-breaking sector, gauge invariance, i.e., cancellation of gauge anomalies in flat space-time, is not enough to fix the hypercharges. Adding the condition of absence of gravitational anomalies we obtain three possible sets of hypercharges. Physical requirements, such as vector electromagnetism allow one to discard the nonstandard solutions.

In summary, the presence of torsion in a curved spacetime background does not change the standard conditions to cancel the gauge and mixed gauge-gravitational anomalies. However the torsion gives new contributions to the leptonic current anomaly that in principle could lead to new physical effects.

\section{ACKNOWLEDGMENTS}

The authors thank L. Alvarez-Gaumé for useful discussions. We would also like to thank A. Gómez Nicola for his suggestions. This work was partially supported by the Ministerio de Educación y Ciencia (Spain) (CICYT AEN951285-E). 
[1] N. D. Birrell and P. C. W. Davies, Quantum Fields in Curved Space (Cambridge University Press, Cambridge, England, 1982).

[2] F. W. Hehl, P. von der Heyde, G. D. Kerlick, and J. M. Nester, Rev. Mod. Phys. 48, 393 (1976).

[3] P. Ramond, Field Theory (Addison-Wesley, Reading, MA, 1989).

[4] M. Nakahara, Geometry, Topology and Physics (IOP, Berkshire, 1990).

[5] L. L. Buchbinder, S. D. Odintsov, and I. L. Shapiro, Effective Action in Quantum Gravity (IOP, Berkshire, 1992).

[6] K. Fujikawa, Phys. Rev. D 21, 2848 (1980).

[7] S. Yajima, Class. Quantum Grav. 5, L207 (1988).

[8] R. E. Gamboa Saravi, M. A. Muschietti, F. A. Schaposnik, and J. E. Solomin, Ann. Phys. (N.Y.) 157, 360 (1984).

[9] K. Fujikawa, Phys. Rev. D 29, 285 (1984).

[10] A. Andrianov and L. Bonora, Nucl. Phys. B233, 232 (1984).

[11] H. T. Nieh and M. L. Yan, Ann. Phys. (N.Y.) 138, 237 (1982);
W. H. Goldthorpe, Nucl. Phys. B170, 307 (1980).

[12] G. Cognola and P. Giacconi, Phys. Rev. D 39, 2987 (1989); A. P. Balachandran, G. Marmo, V. P. Nair, and C. G. Trahern, ibid. 25, 2713 (1982); G. Cognola and S. Zerbini, Phys. Lett. B 195, 435 (1987).

[13] L. Ibáñez, Proceedings of the 5th ASI on Techniques and Concepts in High Energy Physics (Plenum, New York, 1989).

[14] J. Minn, J. Kim, and C. Lee, Phys. Rev. D 35, 1872 (1987).

[15] L. N. Chang and H. T. Nieh, Phys. Rev. Lett. 53, 21 (1984); H. T. Nieh, ibid. 53, 2219 (1984); S. Yajima and T. Kimura, Prog. Theor. Phys. 74, 866 (1985).

[16] L. Alvarez-Gaumé and E. Witten, Nucl. Phys. 234, 269 (1983); L. Alvarez-Gaumé and P. Ginsparg, Ann. Phys. (N.Y.) 161, 423 (1985).

[17] L. Caneschi and P. Valtancoli, Nucl. Phys. B258, 540 (1985).

[18] J. A. Minahan, P. Ramond, and R. C. Wagner, Phys. Rev. D 41, 715 (1990).

[19] C. Q. Geng and R. E. Marshak, Phys. Rev. D 39, 693 (1989). 